\title{
EDITORIAL
}

\section{ESCALANDO AS PRÁTICAS ÁGEIS}

\author{
SCALING AGILE PRACTICES
}

\author{
Renato Penha \\ Universidade Nove de Julho (UNINOVE) \\ rp.renatopenha@gmail.com \\ Luciano Ferreira da Silva \\ Universidade Nove de Julho (UNINOVE) \\ Doutorado em Administração na Pontifícia Universidade Católica (PUC-SP) \\ If_silvabr@yahoo.com.br
}

Resumo

A aplicação de práticas ágeis é um desafio para muitas organizações. Mas isso torna-se insuficiente para que toda a organização possa ter a agilidade esperada em todos os seus projetos, aumentando enormemente o grau desse desafio. A existência de diversas práticas e frameworks como Safe, Scrum of Scrum, entre outros, podem trazer dúvidas sobre de que forma e momento essa transformação deverá ocorrer, além de não existir um consenso para isso. Além disso, não basta escolher o método mais apropriado para obterem resultados melhores na adoção do ágil escalado, pois independente do framework escolhido, as organizações precisarão promover mudanças em sua cultura e outros processos gerenciais. Em relação à cultura organizacional, elas deverão se aproximar e empoderar seus stakeholders, evitando a concorrência de seus projetos e passando a priorizar as entregar de maior valor aos seus negócios. Em relação aos processos gerenciais, precisará aumentar o suporte aos times, a compartilhar os resultados e possuir pessoas engajadas que disseminem as práticas ágeis por todos os níveis. Internamente, as organizações precisarão revisar suas práticas ágeis, com nossa recomendação de resolver todos os seus problemas atuais, antes de exponenciá-los por meio do ágil escalado. Neste contexto, este comentário editorial visa contribuir com a discussão sobre como escalar as práticas ágeis.

Palavras-chave: Ágil. Ágil escalado Frameworks Ágeis. Práticas Ágeis. Cultura organizacional.

Abstract

The application of agile practices is a challenge for many organizations. However, this becomes insufficient to achieve the agility expected in all projects of the organization, significantly increasing the degree of this challenge. The existence of several practices and frameworks such as Safe, Scrum of Scrum, among others, may raise doubts about how and when this transformation should occur. Also, there are several practices and frameworks, such as Safe, Scrum of Scrum, which bring a lack of consensus for what framework is more appropriate. Besides, it is not enough to choose the most appropriate method to obtain better results in the adoption of agile scaling, because regardless of the chosen framework, organizations will need to promote changes in their culture and other management processes. Regardless of the chosen framework, to obtain better results in the adoption of agile scaling, organizations will need to promote changes in their culture and management processes. In relation to concerning the organizational culture, they should approach and empower their stakeholders, avoiding competition from their projects, and starting to prioritize delivering the most value to their business. About management processes, you will need to increase support for teams, share results, and have engaged people who disseminate agile practices at all levels. Internally, organizations will need to review their agile practices, and the recommendation will be to resolve all their current problems before exposing them through agile escalation. In this context, this editorial comment aims to contribute to the discussion on how to scale agile practices.

Keywords: Agile. Agile scaled. Agile Frameworks. Agile Practices. Organizational culture.

\section{Cite como - American Psychological Association (APA)}

Penha, R., Silva, L. F. da, \& Russo, R. de F. S. G. (2020, maio/ago.). Escalando as práticas ágeis. Editorial. Revista de Gestao e Projetos (GeP), 11(2), 1-11. https://doi.org/10.5585/gep.v11i2.18133. 


\section{Introdução}

$\mathrm{O}$ gerenciamento de projetos e as metodologias ágeis estão cada vez mais se popularizando entre profissionais de ramos diversos. Em artigo da revista Exame, Granato, Bomfim e Branco (2020) exploram como agentes públicos conseguiram obter benefícios da utilização de metodologias ágeis. Em reportagem da Gazeta do Povo, Rupp (2020) descreve como negócios de gastronomia podem se beneficiar da metodologia ágil para promoção da inovação.

Em se tratando dos desafios, devemos ter em mente que cada organização é única, possuindo suas particularidades no tocante à cultura e aos processos, tornando-a diferente das demais. Assim, cada vez mais se torna necessário identificar a capacidade das organizações no processo de adaptação das práticas ágeis, compreendendo também quais alterações e impactos são decorrentes dessas ações no gerenciamento dos projetos (Serrador \& Pinto, 2015). Portanto, durante o processo de mudança gerencial para o ágil, as organizações devem optar entre adotar um framework existente ou adaptá-lo ao seu ambiente e valores.

No contexto de introdução das práticas de gerenciamento de projetos, quando o tema Gerenciamento de Projetos e Transformação Digital começam a andar lado a lado nas empresas, o tema agilidade ganha um destaque ainda maior. Apesar dos temas estarem de certa forma relacionados e convergirem em muitas situações, devemos ter em mente que não se trata da mesma coisa. Para uma empresa se tornar ágil, ela não precisa ser digital, porém, para ser digital, ela precisa se tornar ágil. Neste contexto, se torna necessário compreender que essa relação pode não ser uma tarefa tão simples. As empresas devem estar preparadas estrategicamente em relação aos seus processos gerenciais, devendo abranger todos os times responsáveis pelo desenvolvimento dos projetos e produtos (Abrar et al., 2019; Dingsøyr, Falessi, \& Power, 2019).

Mesmo com a implantação das práticas ágeis, as organizações para se manterem competitivas se deparam com o desafio de fornecer soluções aderentes às necessidades voláteis dos stakeholders. Para ultrapassar essa barreira, as organizações estão apoiando os times de desenvolvimento, mudando seus processos internos para adotar a agilidade como facilitador e impulsionador estratégico de seus projetos. Assim, o objetivo de escalar as práticas ágeis é almejado pela organização a fim de alinhar as ações de mudança rumo aos alvos estratégicos.

Para Ebert e Paasivaara (2017), o ágil escalado é representado pela capacidade em gerar agilidade presente no nível do time de desenvolvimento, adotando as mesmas práticas, princípios em outros níveis da organização. Para Dingsøyr, Falessi e Power (2019), os frameworks ágeis escalados, podem lidar com muitas equipes, grandes ou não, trabalhando ao mesmo tempo em um projeto. Assim, por exemplo, no processo de 
transformação digital, o relacionamento entre as pessoas e as ferramentas de apoio ao gerenciamento dos projetos possui um papel de destaque no aperfeiçoamento da capacidade da organização de executar, de acordo com sua estratégia, a proposta de escalar as práticas ágeis (Amjad et al., 2017). Neste sentido as mudanças propostas ajudarão a descentralizar o processo de tomada de decisão dos projetos, criando maior transparência na relação com os stakeholders (Conboy \& Carroll, 2019). Processos escalados também permitirão aumento no desempenho operacional ao mesmo tempo em que os valores ágeis são incorporados aos valores da organização (Iivari \& Iivari, 2011).

Para obterem sucesso nesse processo, as organizações devem possuir um plano de mudança claro e aderente à sua estratégia organizacional, caso contrário, o insucesso na tentativa de adoção do ágil escalado pode resultar na insatisfação dos stakeholders, na perda financeira e de participação no mercado (Felipe et al., 2017). Com base neste contexto, este comentário editorial tem como objetivo descrever aspectos relacionados a escalar as práticas ágeis. Para se ter ideia da dimensão desse cenário, a pesquisa gerada pelo 14th Annual State of Agile Report (2020) indicou que as empresas ao redor do mundo, quando perguntadas sobre quais os principais indicadores utilizados para acompanhamento da evolução da transformação ágil, citaram satisfação do cliente e valor comercial como sendo fatores mais importantes do que prazo da entrega e produtividade.

Uma organização quando inicia a escalada de suas práticas ágeis pode ter parte da equipe praticando ágil e parte utilizando técnicas e ferramentas tradicionais de gerenciamento de projetos. O que elas devem ter em mente é que escalar as práticas ágeis não é uma tarefa fácil e não acontecerá em um curto espaço de tempo.

Existem vários tipos de frameworks de ágil escalado no mercado, como o Scaled Agile Framework (SAFe), Large Scale Scrum (LeSS) e Scrum of Scrums. O ponto em comum para adoção de qualquer deles referese à necessidade de adaptação ao que a organização está vivenciando no momento de pensar no processo de escalar as práticas ágeis. $\mathrm{Na}$ maioria das vezes, as iniciativas de métodos ágeis tendem a ocorrer inicialmente nos times (bottom up) e depois os resultados são apresentados à alta gestão, e a decisão de escalar as práticas para outros níveis da organização é tomada. Independente do framework, o que devemos ter em mente é que o objetivo final é executar a estratégia, não escalar o processo.

\section{2 Ágil escalado nas organizações: como se preparar e quais as dificuldades?}

Uma das formas mais eficazes de promover agilidade é por meio da imersão dos processos e práticas gerenciais ágeis alinhadas à estratégia das organizações (Kettunen, 2009). Porém, alguns desafios emergem quando as organizações pretendem escalar as práticas 
ágeis. Dentre eles, Silva et al. (2019) destacam as incertezas e a ambiguidade existentes no gerenciamento dos projetos. Em consonância com esses desafios o 14th Annual State of Agile Report (2020) aponta a cultura organizacional como um dos maiores desafios para adotar e escalar as práticas ágeis. Com relação aos aspectos da cultura organizacional, o relatório destaca a resistência à mudança como o principal desafio a ser superado pelas organizações no processo de escalada ágil, com $48 \%$ do resultado. Outros dois desafios que ganham destaque são a baixa participação da liderança com $46 \%$, e os processos e práticas inconsistentes entre os times com $45 \%$ dos resultados - vale salientar que os respondentes tiveram a oportunidade de selecionarem múltiplas opções nas respostas da pesquisa.

Partindo para a influência que as organizações podem sofrer durante o processo de escalada ágil, os stakeholders surgem como um fator interno e externo, possuindo relação direta no ciclo de vida dos projetos. Stakeholders externos são caracterizados por clientes, setores do governo ou fornecedores. Os stakeholders internos são considerados os recursos humanos da organização, que agregam valor aos projetos por meio de suas capacidades e competências (Kutomi \&
Piscopo, 2013; Obradovic et al., 2016). Para elucidar tais influências, Pichler (2020) destaca três grandes dificuldades a serem ultrapassadas pelas organizações durante a implantação do ágil escalado. A primeira refere-se ao engajamento e a participação efetiva dos stakeholders externos durante todas as fases do projeto, minimizando possíveis ruídos que possam ter impacto negativo durante a fase de escalada. A segunda dificuldade está associada ao empoderamento dos stakeholders (Pichler, 2020), pois a partir do momento em que os stakeholders se empoderam em relação aos processos organizacionais e desenvolvem conhecimentos claros sobre os objetivos, eles evitam a concorrência de entrega de seus projetos, passando a priorizar as entregas de maior valor aos seus negócios. Por fim, a terceira dificuldade está associada à mudança cultural da organização em relação com os envolvidos nos projetos, e superar esse desafio exigirá grande esforço de todos os times distribuídos entre todos os níveis da organização. Para o autor, a prioridade da organização durante o processo de escalada ágil deverá ser satisfazer o stakeholder, tanto interno como externo. A Figura 1 sintetiza as principais dificuldades a serem enfrentadas pelas organizações durante a escalada das práticas ágeis. 
Figura 1 - Dificuldades para a adoção do ágil escalado nas organizações

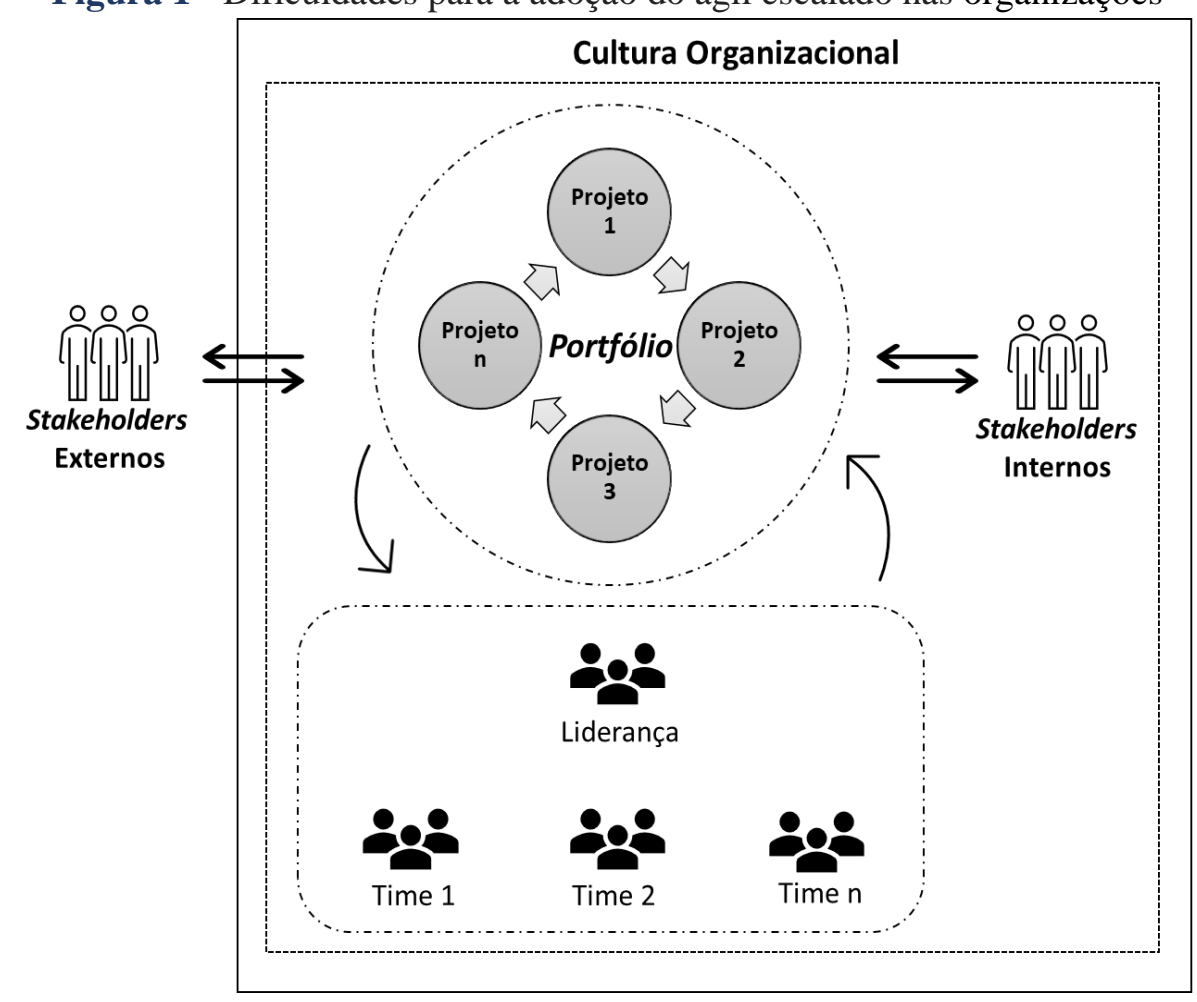

Fonte: Elaborado pelos autores.

Em se tratando de escalar as práticas ágeis, as organizações devem estar preparadas estrategicamente quanto à abordagem de seus times de desenvolvimento. Tais processos devem abranger não só o time de desenvolvimento, mas também o gerenciamento de portfólio de seus projetos (Conboy \& Carroll 2019). Em organizações nas quais as práticas ágeis não estão disseminadas entre o gerenciamento de portfólio de projetos, os times ágeis podem encontrar dificuldades em mensurar o real desempenho de entrega dos projetos (Sweetman \& Conboy 2019). Aqui vale destacar que a equipe de gerenciamento de portfólio é responsável pela priorização do backlog das atividades e da alocação dos recursos, com destaque para os recursos humanos, ficando a cargo das equipes ágeis a entrega dos projetos (Turetken, Stojanov, \& Trienekens 2017). Nesse sentido, manter a estabilidade dos modelos clássicos e a flexibilidade dos modelos ágeis surge como uma solução de gestão e escala dos times ágeis (Conforto \& Amaral, 2016).

Portanto, ao tomar consciência do impacto da cultura organizacional e da relação do processo de escalar as práticas ágeis com os stakeholders, a organização terá feedback constante dos processos e da execução dos projetos, podendo assim obter informações essenciais para que a liderança possa agir proativamente no apoio e disseminação do ágil escalado. O desafio consistirá em satisfazer os stakeholders sem permitir que eles exerçam influência negativa nos resultados dos projetos 
e na disseminação das práticas que envolvem o ágil escalado, criando uma possível concorrência entre todos os envolvidos e os projetos em andamento.

\section{Quais os frameworks existentes para se escalar as práticas ágeis?}

A adoção de práticas ágeis em escala poderá levar as organizações a desenvolverem sistemas otimizados para apoio aos negócios de maneira disruptiva e diretamente associados à cultura e ao ecossistema de atuação. Para isso, na fase de planejamento será necessário obter o suporte executivo, além de um time motivado e confiante nos princípios, valores e práticas ágeis que sustentam a maneira de produzir resultados (Dingsøyr, Falessi, \& Power, 2019). Em relação aos valores que contribuem para a adoção das práticas ágeis escaladas, pode-se destacar o alinhamento dos times envolvidos nos projetos com a estratégia organizacional. Outro valor determinante é a transparência em todos os níveis da organização, permitindo assim que a comunicação e as opiniões de todos os times possuam o mesmo peso nas decisões estratégicas sem sofrer nenhum tipo de retaliação (Scaled agile, 2020).

Não existe uma receita para apoio nesse processo de construção de valores, porém alguns frameworks podem apoiar as organizações a obterem o maior engajamento das pessoas nessa adaptação. Neste sentido, a organização precisa ter certeza do momento adequado para início da escalada. Para se ter uma noção da criticidade envolvendo essa decisão, o PMI (2017) indica que, caso uma abordagem ágil usada com uma equipe não seja bem sucedida, devemos parar a iniciativa e, imediatamente, analisar e tratar os impedimentos que atrapalham o funcionamento das equipes de forma ágil. Assim, somente depois dessa tratativa as organizações devem voltar e escalar para ampliar ainda mais a adoção da agilidade. Para apoiar essa decisão, Terentim e Gonçalves (2020) citam alguns fatores que devem ser levados em consideração:

(i) Suporte aos times: os times devem possuir a motivação e o engajamento das práticas ágeis atuais, para que no processo de transformação para o ágil escalado, as novas diretrizes possam ser compreendidas e aprimoradas. Desse modo, os times passarão a contribuir para disseminar as práticas para todos os níveis da organização com maior intensidade;

(ii) Compartilhamento dos resultados: com o suporte dos times, os resultados da adoção do ágil escalado alcançará maior atenção dos executivos, obtendo assim como resultado uma abordagem positiva do processo na cultura da organização. Além disso, o compartilhamento dos resultados ajudará na troca de experiências e a manter uma opinião única e favorável às práticas ágeis entre os times;

(iii) Voluntariado: o papel do espírito voluntário pode contribuir na disseminação do uso das práticas ágeis 
em times com maior experiência. $\mathrm{O}$ objetivo é apoiar a capacitação de pessoas de times de desenvolvimento distintos, dando suporte ao amadurecimento ágil em todos os níveis da organização.

Somente após terem ciência desses fatores e a relação com sua cultura e valores, as organizações estarão preparadas para a escolha do framework a ser adotado para apoio ao processo de escalar as práticas ágeis. O 14th Annual State of Agile Report (2020) destaca os principais frameworks utilizados nas empresas que suportam o ágil escalado, representados na Figura 2.

Figura 2 - Frameworks mais utilizados para adoção do ágil escalado

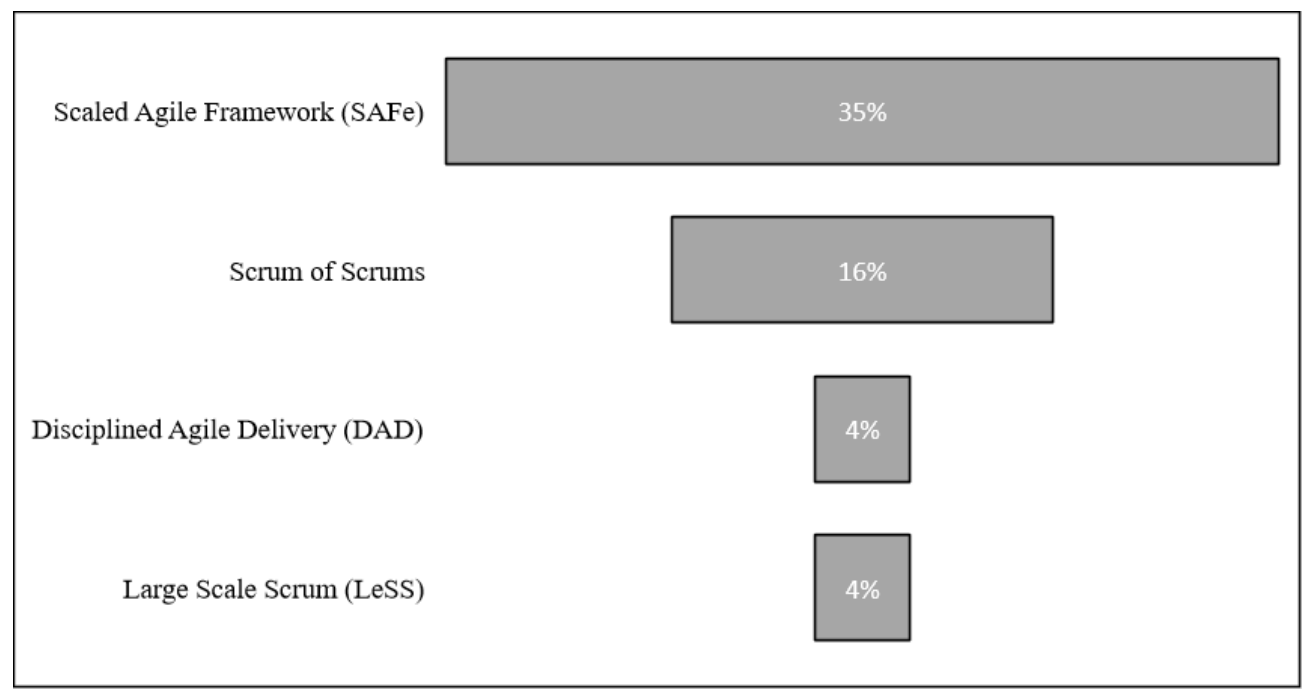

Fonte: Elaborado pelos autores com base no 14th Annual State of Agile Report

O SAFe com $35 \%$ dos resultados é o framework mais adotado pelas organizações, um percentual superior ao dobro do Scrum of Scrums, o segundo framework mais utilizado com $16 \%$ dos resultados. Na sequência e empatados na terceira posição com $4 \%$ dos resultados, estão os frameworks Disciplined
Agile Delivery (DAD) e Large Scale Scrum (LeSS). Para melhor compreensão de cada framework, a Figura 3 destaca os quatro frameworks mais utilizados e as respectivas características para uso nas organizações em busca do sucesso para a escalada ágil. 
Figura 3 - Frameworks para adoção do ágil escalado

\begin{tabular}{|c|c|}
\hline Framework & Descrição \\
\hline $\begin{array}{c}\text { Scaled Agile. SAFe. } \\
\text { Disponível em: } \\
\text { https://www.scaledagilefram } \\
\text { ework.com/safe-core-values/ }\end{array}$ & $\begin{array}{l}\text { Sugerido para organizações de médio e grande porte, é considerado um dos } \\
\text { frameworks de escalada ágil mais utilizados no mundo, possuindo grande } \\
\text { aceitação pelos indivíduos. O framework sugere um roadmap baseado em } 12 \\
\text { passos: (1) posicione o time no ponto de decisão, (2) treine os agentes de } \\
\text { mudança ágil, (3) treine executivos, gerentes e líderes; (4) crie um centro de } \\
\text { excelência ágil, (5) identifique as Cadeias de Valores (Value Streams) e os Agile } \\
\text { Release Train (ART), (6) crie um plano de implementação, (7) prepare-se para o } \\
\text { ART; (8) lance os times dos trens e lance um ART; (9) realize coaching na } \\
\text { execução do ART, (10) lance mais ARTs e os Value Streams; (11) estenda ao } \\
\text { portfólio dos projetos, (12) acelere; }\end{array}$ \\
\hline $\begin{array}{l}\begin{array}{l}\text { Scaled Agile. DAD. } \\
\text { Disponível em: }\end{array} \\
\text { https://www.pmi.org/discipli } \\
\frac{\text { ned- }}{\text { agile/process/introduction- }} \\
\text { to-dad\#Hybrid/ }\end{array}$ & $\begin{array}{l}\text { O DAD é considerado como uma ferramenta de abordagem híbrida no processo } \\
\text { de escada das práticas ágeis. Para isso, baseia-se no aprendizado dos times, } \\
\text { apoiando pessoas a determinarem o melhor maneira de realizarem suas } \\
\text { atividades. } \\
\text { Sua essência possui } 8 \text { princípios primordiais da Agilidade, partindo da premissa } \\
\text { de que é preciso encantar o cliente e que o contexto importa, além de contar ciclos } \\
\text { de vida diferentes, de acordo com a abordagem a ser escolhida. Desse modo, } \\
\text { possui um papel de orientação ao invés de adotar um modelo que serve para toda } \\
\text { a organização. }\end{array}$ \\
\hline $\begin{array}{c}\text { Scaled Agile. Scrum of } \\
\text { Scrums. } \\
\text { Disponível em: } \\
\text { https://www.agilest.org/ente } \\
\text { rprise-transformation/ }\end{array}$ & $\begin{array}{l}\text { Adotado em sua maioria por organizações que já possuem as práticas Scrum. O } \\
\text { framework destaca os papéis e práticas do Scrum, envolvendo mais de um time } \\
\text { nas cerimônias e artefatos. Em se tratando do gerenciamento interno dos } \\
\text { processos, mantém as premissas em relação aos manuseios dos backlogs (produto } \\
\text { e sprint). Porém, para implementação no processo de escala, o framework requer } \\
\text { mudanças na estrutura organizacional em relação ao monitoramento e controle } \\
\text { dos processos. }\end{array}$ \\
\hline $\begin{array}{c}\begin{array}{c}\text { Scaled Agile. LeSS. } \\
\text { Disponível em: }\end{array} \\
\text { https://less.works/pt/less/fra } \\
\text { mework/index }\end{array}$ & $\begin{array}{l}\text { Destaca a mudança contínua e através de experimentação, promovendo } \\
\text { consequentemente uma mudança no status quo e com um foco no produto como } \\
\text { um todo, não em parte. Para que sua adoção tenha sucesso, é necessário porém } \\
\text { que haja uma comunicação consistente e transversal sobre: (1) a intenção de } \\
\text { adotá-lo; (2) o compromisso de que devem existir mudanças estruturais, } \\
\text { adaptando a realidade aos frutos da transformação; e que (3) deverá haver } \\
\text { treinamento e coaching necessários em todos os grupos, principalmente através } \\
\text { de voluntários dos times. }\end{array}$ \\
\hline
\end{tabular}

Fonte: Elaborado pelos autores.

Independente do framework a ser adotado, serão necessários o engajamento e a redução no conflito de interesses entre todos os níveis hierárquicos (Rigby et al., 2018). As 
pessoas responsáveis pelo emprego e divulgação das práticas do framework adotado deverão ter em mente a essência e a simplicidade nos processos ágeis, e que deverão ser utilizados para quebrar os paradigmas contra as práticas ágeis que possam existir em alguns times da organização. Nessa fase, essas pessoas devem se transformar em agentes de transformação, promovendo a cultura da agilidade em todas as esferas da organização, aumentando assim a visibilidade e o formato adequado para as práticas do ágil escalado.

\section{Considerações finais}

Ao final deste comentário editorial destacamos que existem diversos frameworks disponíveis no mercado para escalar as práticas ágeis. $\mathrm{O}$ ponto em comum entre eles refere-se à necessidade de adaptação da organização no momento de planejar e se preparar para adoção do ágil escalado. Na maioria das vezes, as iniciativas tendem a ocorrer inicialmente nos times de maneira bottom up, e depois, os resultados são compartilhados pela organização até a alta gerência. Alguns pontos importantes nessa jornada devem fazer parte do processo, como a necessidade de mudança cultural; ações motivadoras para adoção do ágil em todos os níveis organizacionais e desmitificar a ilusão que isso vai acarretar mudanças na estrutura organizacional. A falha em copiar algo que funcionou para outros é que, junto com possíveis benefícios, os problemas também são migrados e, como não fazem parte da cultura da organização, podem trazer descrédito e novas barreiras dificultando o processo de escalada ágil.

Após a escolha de um framework, compreender e estreitar o relacionamento com os stakeholders internos e externos se torna para as organizações um fator de sucesso no processo de adoção do ágil escalado. É notória a relação que esses stakeholders exercem no portfólio de projetos e mantê-los engajados e mais próximos das tomadas de decisões podem contribuir para minimizar possíveis disputas sobre prioridades de projetos e dos times de desenvolvimento. Desse modo, o nível de confiança dos stakeholders aumenta, gerando assim maior transparência na gestão do portfólio dos projetos. Alinhado com esse pensamento, vale destacar que os times envolvidos no processo de ágil escalado precisam de maior autonomia e habilidades para exercerem suas atividades, sempre suportadas por uma liderança apoiadora e participativa.

Em se tratando do portfólio de projetos, as práticas ágeis em escala permitem que a organização trabalhe seu portfólio com maior precisão. O que isso quer dizer? Com a maior proximidade dos stakeholders nas decisões dos projetos, poderá haver um aumento da efetividade da entrega de valor por meio dos resultados dos projetos. Em poucas palavras, ampliar a cultura de colaboração entre stakeholders e o portfólio de projetos contribuem para que as organizações estejam sempre condicionadas para agir rapidamente às 
possíveis mudanças e promoverem adaptações no seu processo de escalar as práticas ágeis.

Independente do framework de ágil escalado a ser adotado pelas empresas, é necessário adequá-lo à cultura da empresa, aos seus valores e princípios, além de envolver os níveis organizacionais, pois iniciativas top down tendem a ter mais sucesso se todos entenderem os benefícios e responsabilidades pertinentes ao mindset ágil. Princípios ágeis como "fazer o mais rápido possível" podem se tornar em se "fazer o mais do mesmo o mais rápido possível", refletindo diretamente no desempenho dos times. Assim, o planejamento dos projetos e o foco dos times ágeis por todo o ciclo de vida dos projetos, passam a ser fatores essenciais para organizações que desejam escalar os times ágeis. Desse modo, uma questão merece atenção: Não confundir quantidade de times ágeis com times ágeis escaláveis!

Caso as organizações não tenham atenção a esse ponto, problemas comuns das práticas tradicionais de gerenciamentos podem surgir com a aplicação do ágil escalado, com o Resource-Constrained Project Scheduling Problem (Problema de Programação de Projetos com Restrição de Recursos), inexistente em times ágeis não escalados. Não existe uma fórmula ou receita que simplifique as organizações a tomarem a decisão de partirem para a escalada ágil, porém, na mesma proporção que elas desejam aumentar o alcance das práticas ágeis, elas devem aumentar seu foco em sua cultura e valores aplicados à gestão de pessoas, pois as pessoas representam o componente-chave do sucesso na disseminação e na condução do ágil em escala em seus projetos, atendendo assim às necessidades de seus stakeholders.

\section{Referências}

Abrar, M. F., Khan, M. S., Ali, S., Ali, U., Majeed, M. F., Ali, A., ... \& Rasheed, N. (2019). Motivators for large-scale agile adoption from management perspective: A systematic literature review. IEEE Access, 7, 22660-22674.

https://doi.org/10.1109/ACCESS.2019.289621 $\underline{2}$

Amjad, S., Ahmad, N., Saba, T., Anjum, A., Manzoor, U., Balubaid, M. A., \& Malik, S. U. R. (2017). Calculating completeness of agile scope in scaled agile development. IEEE Access, 6, 5822-5847. https://doi.org/10.1109/ACCESS.2017.276535 $\underline{1}$

Conboy, K., \& Carroll, N. (2019). Implementing large-scale agile frameworks: challenges and recommendations. IEEE Software, 36(2), 4450. https://doi.org/10.1109/MS.2018.2884865

Conforto, E. C., \& Amaral, D. C. (2016). Agile project management and stage-gate model-A hybrid framework for technology-based companies. Journal of Engineering and Technology Management, 40, 1-14. https://doi.org/10.1016/j.jengtecman.2016.02. $\underline{003}$

Dingsøyr, T., Falessi, D., \& Power, K. (2019). Agile development at scale: the next frontier. IEEE Software, 36(2), 30-38.

Ebert, C., \& Paasivaara, M. (2017). Scaling agile. IEEE Software, 34(6), 98-103. https://doi.org/10.1109/MS.2017.4121226

Felipe, C. M., Roldán, J. L., \& Leal-Rodríguez, A. L. (2017). Impact of organizational culture values on organizational agility. Sustainability, 9(12), 2354. https://doi.org/10.3390/su9122354 
Fernandez, D. J., \& Fernandez, J. D. (2008). Agile project management-agilism versus traditional approaches. Journal of Computer Information Systems, 49(2), 10-17. https://doi.org/10.1080/08874417.2009.11646 $\underline{044}$

Granato, L., Bomfim, M., \& Branco L. (2020). Ágil e às pressas: metodologia salva equipes do caos na pandemia. Revista Exame online. Recuperado em 25.08.2020 de: https://exame.com/revista-exame/agil-e-aspressas-metodologia-salva-equipes-do-caosna-pandemia/

Iivari, J., \& Iivari, N. (2011). The relationship between organizational culture and the deployment of agile methods. Information and software technology, 53(5), 509-520. https://doi.org/10.1016/j.infsof.2010.10.008

Kettunen, P. (2009). Adopting key lessons from agile manufacturing to agile software product development-A comparative study. Technovation, 29(6-7), 408-422. https://doi.org/10.1016/j.technovation.2008.10 .003

Kutomi, A. S., \& Piscopo, M. R. (2013). Comprometimento e Envolvimento dos Stakeholders Internos em Projetos. Anais do II Simpósio de Gestão de Projetos - II SINGEP, São Paulo, SP, Brasil.

Obradović, V., Kostić, S. C., \& Mitrović, Z. (2016). Rethinking project management-Did we miss marketing management? ProcediaSocial and Behavioral Sciences, 226, 390-397. https://doi.org/10.1016/j.sbspro.2016.06.203

Pichler, R. (2020). How to Lead in Product Management: Practices to Align Stakeholders, Guide Development Teams, and Create Value Together. Pichler Consulting.

Project Management Institute. (2017). Um Guia do Conhecimento de Gerenciamento de Projetos (Guia PMBoK®). (6a.). Newton Square, Pensilvânia: PMI - Project Management Institute.
Rigby, D. K., Sutherland, J., \& Noble, A. (2018). Agile at scale. Harvard Business Review, 96(3), 88-96.

Rupp, I. (2020). Como os negócios de gastronomia podem se beneficiar da metodologia ágil e da inovação. Bom Gourmet - Gazeta do Povo. Recuperado em 25.08.2020 de:

https://www.gazetadopovo.com.br/bomgourm et/hack-pela-gastronomia/como-negocios-degastronomia-podem-se-beneficiar-dametodologia-agil/

Scaled Agile. Portfolio kanban. Recuperado em 25.08.2020 de:

https://www.scaledagileframework.com/portfo lio- kanban/

Serrador, P., \& Pinto, J. K. (2015). Does Agile work? A quantitative analysis of agile project success. International Journal of Project Management, 33(5), 1040-1051. https://doi.org/10.1016/j.ijproman.2015.01.00 $\underline{6}$

Silva, D. S., Ghezzi, A., de Aguiar, R. B., Cortimiglia, M. N., \& ten Caten, C. S. (2019). Lean Startup, Agile Methodologies and Customer Development for business model innovation. International Journal of Entrepreneurial Behavior \& Research. 26(4), 595-628. https://doi.org/10.1108/IJEBR-072019-0425

State of Agile. 14th Annual State of Agile Report (2020). Recuperado em 25.08.2020 de: https://stateofagile.com/

Sweetman, R. and K. Conboy (2019). "Finding the Edge of Chaos: A Complex Adaptive Systems Approach to Information Systems Project Portfolio Management." In: Proceedings of the 27th European Conference on Information Systems (ECIS). StockholmUppsala: Sweden. Recuperado em 25.08.2020 de:

https://aisel.aisnet.org/cgi/viewcontent.cgi?arti $\underline{\text { cle }=1010 \& \text { context }=\text { ecis2019 rip }}$

Terentim, G., \& Gonçalves, V. (2020). Gestão de Mudanças em Abordagens Ágeis: HCMBOK to Agile: The Human Chance Management Body of Knowledge. Rio de Janeiro: Brasport. 